\title{
Metabolic remodelling in heart failure revisited
}

\author{
Miranda Nabben, Joost J. F. P. Luiken and Jan F. C. Glatz
}

The Review by Edoardo Bertero and Christoph Maack (Metabolic remodelling in heart failure. Nat. Rev. Cardiol. 15, 457-470; 2018) ${ }^{1}$ addresses the changes in cardiac energy metabolism that occur during the development of heart failure (HF). The authors convincingly argue that alterations of intermediate substrate metabolism and oxidative stress, rather than an ATP deficit per se, account for maladaptive remodelling and dysfunction. Despite the comprehensive discussion of this topic, some aspects need further clarification or elaboration.

$\mathrm{HF}$ is a clinical syndrome characterized by a myocardial abnormality causing systolic and/or diastolic ventricular dysfunction ${ }^{2}$. The main examples, as discussed in the Review $^{1}$, are pressure overload-induced HF, ischaemic HF, and diabetes-induced HF (diabetic cardiomyopathy). From a metabolic perspective, these forms of $\mathrm{HF}$ differ markedly in that in both pressure overload-induced HF and ischaemic HF, substrate preference shifts towards increased glucose utilization, whereas in diabetic cardiomyopathy, fatty acids become the preferred substrate ${ }^{3,4}$. In the Review, HF is considered to be caused by pressure overload or ischaemia, whereas diabetic cardiomyopathy is discussed separately, which is confusing. For example, the statement that "The failing heart is characterized by an increase in glucose uptake and glycolytic rates" (page 461) ${ }^{1}$ is valid for pressure overload-induced HF and ischaemic HF, not diabetes-induced HF.

Toxic intracellular accumulation of lipid species (lipotoxicity) is mentioned as a contributing cause of HF. However, the contribution of lipotoxicity to the progression of HF is well-known in diabetic HF but has not been established for pressure overload-induced $\mathrm{HF}^{3}$. The same applies for the role of decreased insulin sensitivity in developing $\mathrm{HF}^{4}$. Moreover, lipotoxicity and insulin resistance are unlikely to be general features of the pressure-overloaded heart.

With respect to targeting substrate metabolism as a treatment option for HF, the hypothesis is discussed that inhibition of fatty acid oxidation might be beneficial because it would induce a shift towards increased utilization of glucose, which has higher oxygen efficiency than fatty acids. Such intervention is helpful in ischaemic HF but not in pressure overload-induced $\mathrm{HF}^{5,6}$ and certainly not in diabetic HF because that would lead to a further mismatch between fatty acid uptake and oxidation, resulting in increased intracellular accumulation of toxic lipid species ${ }^{5,7}$.

The interplay between the intracellular utilization of glucose and of fatty acids for oxidative energy provision (the Randle cycle) is adequately described, but the Review does not mention that a major rate-governing kinetic step in overall myocellular glucose utilization is cardiac glucose uptake ${ }^{8}$, and that in fatty acid utilization it is the fatty acid uptake process (that is, trans-sarcolemmal transport) ${ }^{9}$. Specifically, the relative presence of glucose transporters (GLUT1 and GLUT4) and fatty acid transporters (mainly SR-B2, also known as CD36) in the sarcolemma determines the myocardial utilization of glucose and fatty acids, respectively, and, as a corollary, controls cardiac substrate preference. Therefore, increased sarcolemmal CD36 has been found to be an important early hallmark of the development of diabetic $\mathrm{HF}^{9}$. Furthermore, selectively manipulating the recruitment to the sarcolemma of either GLUT4 or CD36 has been reported in experimental animal studies as a suitable approach to rebalance cardiac substrate utilization and improve cardiac contractile function ${ }^{10}$.

Finally, besides discerning between forms of HF, distinction should also be made between stages of HF development because the type and degree of metabolic adaptation of the heart change during the course of HF progression 7 . Monitoring the cardiac metabolic state is, therefore, not only of interest for early identification of changes in substrate preference but also to predict and assess the effectiveness of treatment.

Miranda Nabben*, Joost J. F. P. Luiken and Jan F. C. Glatz

Department of Genetics \& Cell Biology, Maastricht University, Maastricht, The Netherlands. *e-mail:m.nabben@maastrichtuniversit.nl https://doi.org/10.1038/s41569-018-0115-8

1. Bertero, E. \& Maack, C. Metabolic remodelling in heart failure. Nat. Rev. Cardiol. 15, 457-470 (2018).

2. Ponikowski, P. et al. 2016 ESC guidelines for the diagnosis and treatment of acute and chronic heart failure. Eur. Heart J. 37, 21 29-2200 (2016).

3. Stanley, W. C., Recchia, F. A. \& Lopaschuk, G. D. Myocardial substrate metabolism in the normal and failing heart. Physiol. Rev. 85, 1093-1129 (2005).

4. Boudina, S. \& Abel, E. D. Diabetic cardiomyopathy, causes and effects. Rev. Endocr. Metab. Disord. 11, 31-39 (2010)

5. Tuunanen, $\mathrm{H}$. et al. Free fatty acid depletion acutely decreases cardiac work and efficiency in cardiomyopathic heart failure. Circulation 114, 2130-2137 (2006).

6. Umbarawan, Y. et al. Myocardial fatty acid uptake through CD36 is indispensable for sufficient bioenergetic metabolism to prevent progression of pressure overload- induced heart failure. Sci. Rep. 8 , 12035 (2018)

7. Abdurrachim, D. et al. Good and bad consequences of altered fatty acid metabolism in heart failure: evidence from mouse models. Cardiovasc. Res. 106, 194-205 (2015).

8. Savage, D. B., Petersen, K. F \& Shulman, G. I. Disordered lipid metabolism and the pathogenesis of insulin resistance. Physiol. Rev. 87, 507-520 (2007).

9. Glatz, J. F. C. \& Luiken, J. J. F. P. Dynamic role of the transmembrane glycoprotein CD36 (SR-B2) in cellular fatty acid uptake and utilization. J. Lipid Res. 59, 1084-1093 (2018).

10. Yang, J. et al. CD36 deficiency rescues lipotoxic cardiomyopathy. Circ. Res. 100, 1208-1217 (2007).

Competing interests

The authors declare no competing interests.

\section{Reply to 'Metabolic remodelling in heart failure revisited'}

\section{Edoardo Bertero and Christoph Maack}

We thank Nabben and colleagues for their constructive Correspondence (Metabolic remodelling in heart failure revisited. Nat. Rev. Cardiol. https://doi.org/10.1038/ s41569-018-0115-8; 2018) $)^{1}$ on our Review (Metabolic remodelling in heart failure. Nat. Rev. Cardiol. 15, 457-470; 2018) ${ }^{2}$. We agree with their comments and acknowledge that most of these critical points result from the necessity of giving readers a comprehensive overview of a broad area of cardiovascular research within the limited space of a Review article. Here are our responses to their comments.

We decided to discuss diabetic cardiomyopathy separately from pressure overload-induced and ischaemic heart failure (HF) because they differ 